ALIRNATA

Paper ID \#8134

\title{
Virtual Office Hours through Video Conferencing: Lessons Learned
}

Dr. Daniel Dickrell III, University of Florida 


\section{Virtual Office Hours through Video Conferencing: Lessons Learned}

\section{Introduction}

The ability to hold academic office hours via video conferencing technology has been greatly enhanced with the development of widely distributed, low-cost platforms over the last few years. In addition to traditional forms of remote academic communication such as telephone and email, it is now possible to render academic assistance at a high level of effectiveness in both realtime/asynchronous settings across distance using various technical platforms. The educational efficacy of several of these new modern communication tools, and the experiences with them, are discussed here.

\section{Platforms}

The synergistic combination of high-speed broadband internet and small, deployable cameras has greatly improved the ability of students and teachers to have meaningful, real time academic communications over video-conferencing platforms. Here is a brief review of the available platforms and services capable of accomplishing distance-based office hours:

\section{Apple FaceTime}

Apple's FaceTime service is a traditional video-chat service that is integrated into much of Apple's current hardware offerings. It serves as a simple and efficient way of conducting a video-call where the "telepresence" of the participants closely approximates a real-world office communication interaction. FaceTime interactions flow very much like a conversation taking place on a street-corner or informal setting: the level of information exchange is fairly limited to what can be expressed orally with limited ability to draw or depict any visual information.

\section{Microsoft Skype}

The Skype application (now owned by Microsoft) is a popular communication service that was initially voice-only, but now offers a more full-featured video conferencing capability.

Google+ with Hangouts

A relative new service is the Google+ with Hangout application. Google+ is Google's social media platform (analogous to Facebook) but what differentiates itself from the relatively static nature of traditional social media sites (Facebook, Twitter, Tumblr) is the ability through the use of the Hangout feature to do a simultaneous video conference with up to nine other participants. The internet browser based application also allows for any/all of the participants to share their screen (screen-casting). This capability enhances the educational discourse as it allows for the visual exchange of problem information more readily than a normal video-conference call. The 
Hangout on Air feature allows for a mirrored copy of the Hangout proceedings to be available on the user's Youtube video account to be shared privately or publically to participants not able to attend the Hangout in real time.

\section{Citrix GoToWebinar}

GoToWebinar by Citrix is similar to Google+ with Hangouts except the information flow is more uni-directional, with a single speaker conducting a presentation and limited interjection capability from the participating audience. However the live participant number restriction on GoToWebinar (hundreds of participants) is much greater than Google+ with Hangouts (ten participants) due to this restriction and its impact on technical considerations such as video bandwidth usage and moderation. Audience participants in GoToWebinar are able to ask, in a moderated setting, text-based questions via a chat dialog which the presenter can address in realtime or in a delayed question-and-answer format.

\section{Student Experiences}

All of the above platform tools were used in a semester of a large undergraduate engineering mechanics course (enrollment 500 students) for virtual office hours. Participants in these office hours were those who were either unable to attend physical office hours to due schedule conflicts, or those who were not physically able to attend to remote deployment (internships, coops, etc.). Table 1 shows the summarized findings of the platforms over the semester.

The most useful of the tools were Google+ with Hangouts and GoToWebinar due to their cost, student interesting, and ability to screen-cast the instructor's computer in solving problems and illustrating solutions, albeit in different forms. The most enthusiastic of all virtual office hour participants were those interested in Google+ with Hangouts. The theorized cause for this is that there is a small, but fervently passionate subset of engineering students who are excited about the Android operating system (hackers and computer tinkerers) that have embrace the Google+ with Hangout feature as an integral part of the Android ecosystem.

FaceTime and Skype were regarded as less useful and more gimmicky in a way that did not contribute to the overall learning goal since their experience could be closely approximated by a traditional phone call. Skype was very popular among interested participants, yet it failed to produce useful and meaningful academic interactions. FaceTime in particular failed as a useful tool for virtual office hours due to overwhelming lack of student awareness or interest. Students who were open to video-conferenced office hours almost universally opted for Skype or Google+ with Hangouts, despite the majority of the student users having Apple hardware (laptops, desktops, phones, tablets) available to them for which many have the FaceTime application installed by default.

\section{Instructor Experiences}


The platforms discussed were used by both principal instructor and teaching assistants to supplement their traditional office hours. From an instructional standpoint the collective user experience closely mirrored that of the students': the ability to screen-cast information was of paramount importance to useful virtual office hours. To differentiate the screen-cast capable applications (Google+ and GoToWebinar), the most appropriate platform for a traditional "office hour experience" was Google+ with Hangout where the exchange of information between student and teacher was necessary. GoToWebinar was most useful for exam reviews and other large-scale gatherings of student participants where the uni-directionality of information flow was most appropriate.

\section{Conclusions}

Of the low-cost, widely-available video conferencing platforms discussed for virtual office hour use it seemed that Google+ with Hangouts and GoToWebinar were most useful to both students and instructors: Google+ with Hangouts for virtual office hours and GoToWebinar for large exam reviews. This is not to say that FaceTime or Skype are not without merit, as more seasoned users of either platform are perhaps capable of more than the authors of this paper managed to achieve. Overall the experience with these new technical methods of rendering academic aid from all involved, students and instructors was a positive one and meritorious of future study. 


\begin{tabular}{|c|c|c|c|c|}
\hline Application & $\begin{array}{c}\text { Apple } \\
\text { FaceTime }\end{array}$ & $\begin{array}{c}\text { Microsoft } \\
\text { Skype }\end{array}$ & $\begin{array}{c}\text { Google+ } \\
\text { with Hangouts }\end{array}$ & $\begin{array}{c}\text { Citrix } \\
\text { GoToWebinar }\end{array}$ \\
\hline $\begin{array}{c}\text { Simultaneous } \\
\text { Users }\end{array}$ & 2 & 2 & 10 & $\sim 100$ \\
\hline $\begin{array}{c}\text { Screen-casting } \\
\text { Capability }\end{array}$ & $\mathrm{X}$ & $\mathrm{X}$ & $\sqrt{ }$ & $\sqrt{ }$ \\
\hline $\begin{array}{c}\text { Audio-Video } \\
\text { Transmission }\end{array}$ & bi-directional & bi-directional & bi-directional & one-way \\
\hline Cost & $\$ 0.99$ & free & free & free \\
\hline $\begin{array}{c}\text { Student } \\
\text { Interest }\end{array}$ & low & high & high & medium \\
\hline
\end{tabular}

Table 1. Summarization table of virtual office hour interaction platforms used during the semester 